\title{
Flexible endoscope visualization to assist in the removal of a string of 10 schwannomas at the cauda equina: technical case report
}

\author{
Yu-Cheng Ren, MD, ${ }^{1}$ Bin-Jie Zhao, MD,, Zhi-Yi Xie, MD,' Guang-Yu Ying, MD, \\ Fang Shen, MD, PhD, ${ }^{2}$ and Yong-Jian Zhu, MD, PhD ${ }^{1}$ \\ 'Department of Neurosurgery, The Second Affiliated Hospital of Zhejiang University School of Medicine, Hangzhou, Zhejiang, \\ China; and ${ }^{2}$ Emergency Department, Latrobe Regional Hospital, Traralgon, Victoria, Australia
}

\begin{abstract}
Bead-like schwannomas at the cauda equina are rare but benign intraspinal tumors. They can involve multiple nerve roots and spread within the spinal canal, and open resection would cause significant trauma. The authors have successfully applied a novel minimally invasive technique for the total removal of such schwannomas. A 68-year-old woman presented with a 1-month history of left waist and leg pain. MRI demonstrated multiple intraspinal lesions located from $\mathrm{L} 1$ to S1. The diagnosis was bead-like schwannomas at the cauda equina. Two incisions were made at the T12 and L5 levels. A flexible endoscope was introduced into the spinal canal following hemisemilaminectomy under a microscope to identify the relationship between the tumors and the carrying nerves. After dissecting both cranial and caudal ends of the carrying nerve, the string of bead-like tumors was gently pulled out from the caudal end as a whole. The endoscope was reintroduced into the spinal canal to ensure complete tumor removal. The patient recovered quickly, and no tumor residual was found at postoperative MRI. Flexible endoscope-assisted visualization plus microscopic hemisemilaminectomy via 2 incisions is a feasible minimally invasive approach for selected patients with bead-like schwannomas at the cauda equina.
\end{abstract}

https://thejns.org/doi/abs/10.3171/2020.4.SPINE191390

KEYWORDS bead-like schwannomas; cauda equina; hemisemilaminectomy; flexible endoscope; microscope; surgical technique

0 CHWANNOMAS, one of the most common tumors in the spinal canal, ${ }^{1,2}$ rarely form bead-like shapes on a single carrying nerve root. ${ }^{3,4}$ Bead-like schwannomas of the cauda equina usually grow longitudinally in the lumbar canal. Conventional approaches, such as laminectomy and hemilaminectomy, will interrupt the spinal integrity by removal of bony and connective tissues, leading to extensive tissue trauma and prolonged wound healing. Here we present the first patient with a string of bead-like schwannomas of the cauda equina in whom a flexible endoscope was introduced during surgery to visualize the tumor and identity the exiting nerve root. Total tumor removal was achieved via hemisemilaminectomy under the operating microscope.

\section{Technical Case Report}

A 68-year-old woman presented with a 1-month history of stabbing pain in the left waist radiating to the left lower limb, which was aggravated by coughing. Physical examination found no positive neurological signs. A lumbar contrast-enhanced MRI study demonstrated multiple nodular masses extending from the L1 to S1 level inside the spinal canal (Fig. 1A-C). Furthermore, imaging by FIESTA sequence indicated that the nodules originated from a single nerve root (Fig. 1D). All these findings supported the diagnosis of a string of bead-like schwannomas of the cauda equina. To achieve both minimal invasiveness and optimal tumor exposure, volume rendering technique (VRT) 3D reconstruction of the lumbar CT was performed preoperatively for measurement of the interlaminar spaces and bone windows design. A flexible endoscope was planned to be used intraoperatively to reduce tissue injury because it allows direct visualization of the intraspinal lesion through the small incision. Approval was obtained from the local ethics committee to perform this operation, and 

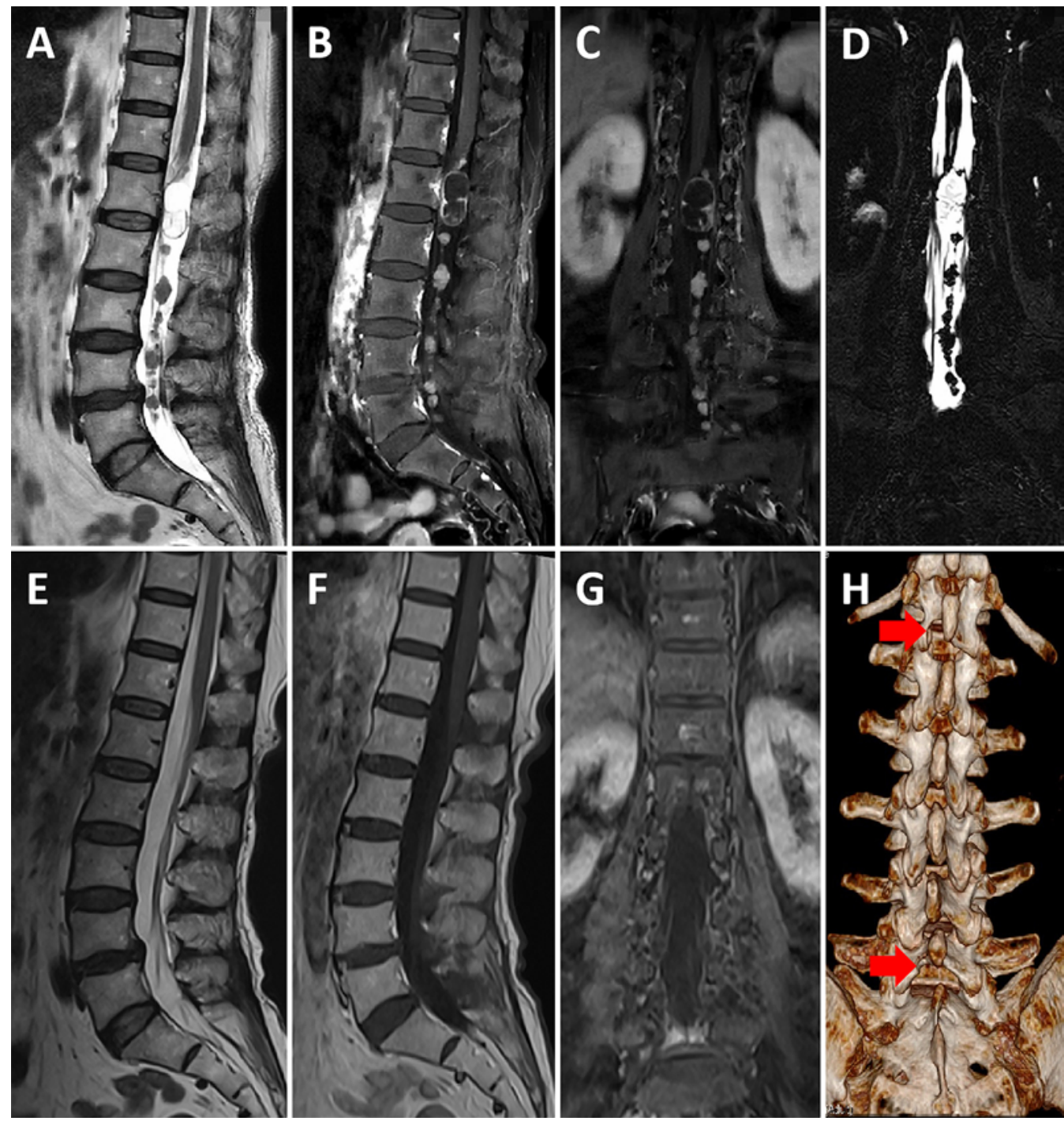

FIG. 1. Imaging studies of the patient's thoracic and lumbar spine. A-D: Preoperative lumbar enhanced MRI with FIESTA sequence demonstrated bead-like schwannomas of the cauda equina (A, sagittal T2-weighted; $B$, sagittal contrast-enhanced T1-weighted; C, coronal FIESTA image; D, coronal contrast-enhanced T1-weighted). E-G: Nine-month postoperative lumbar MRI demonstrated neither tumor residual in the lumbar canal nor lumbar spinal deformity after tumor resection (E, sagittal T2-weighted; F, sagittal contrast-enhanced T1-weighted; G, coronal contrast-enhanced T1-weighted). H: Postoperative day 3 VRT 3D reconstruction of lumbar CT showed the bone windows created for the procedure (red arrows). Figure is available in color online only.

our patient has given informed consent about this novel surgical approach: hemisemilaminectomy at 2 incisions assisted by a flexible endoscope for visualization.

Electrophysiological monitoring was used in this case. After general anesthesia was administered, the patient was placed prone. A 5-cm median incision was made over the spinal processes of T12 and L1, followed by hemisemilaminectomy on the left lower part of the T12 lamina. The bone window was first enlarged to approximately $1.5 \mathrm{~cm} \times$ $1.0 \mathrm{~cm}$, and then the spinal dura mater and arachnoid were sequentially exposed and opened to expose the tumor on the cranial end. The biggest, yellowish, cystic tumor was cut open first to release the cystic fluid and gain room for further exposure along the same carrying nerve root. After another solid tumor was identified, we realized we had reached the caudal end of these bead-like tumors accord- ing to the preoperative imaging study. The tumor-carrying nerve root was cut off at this cranial side of the solid tumor and tied to a suture to avoid slipping into the canal. The second incision was then made above the spinal processes of L5 and S1.

Similarly, hemisemilaminectomy of the left lower part of the L5 lamina was performed, and the dorsal sensory root of the L5 spinal nerve was identified as the tumorcarrying nerve. A flexible endoscope was inserted toward cephalad into the opened dural space via this L5/S1 incision. All 10 tumors, from the caudal end to the cranial end suture, were identified under the endoscope as located on the same nerve root with little adhesion to adjacent cauda equina nerves (Fig. 2). After withdrawing the flexible endoscope, the tumor-carrying nerve root was cut off at the caudal end of the tumors. After dissection of the distal 

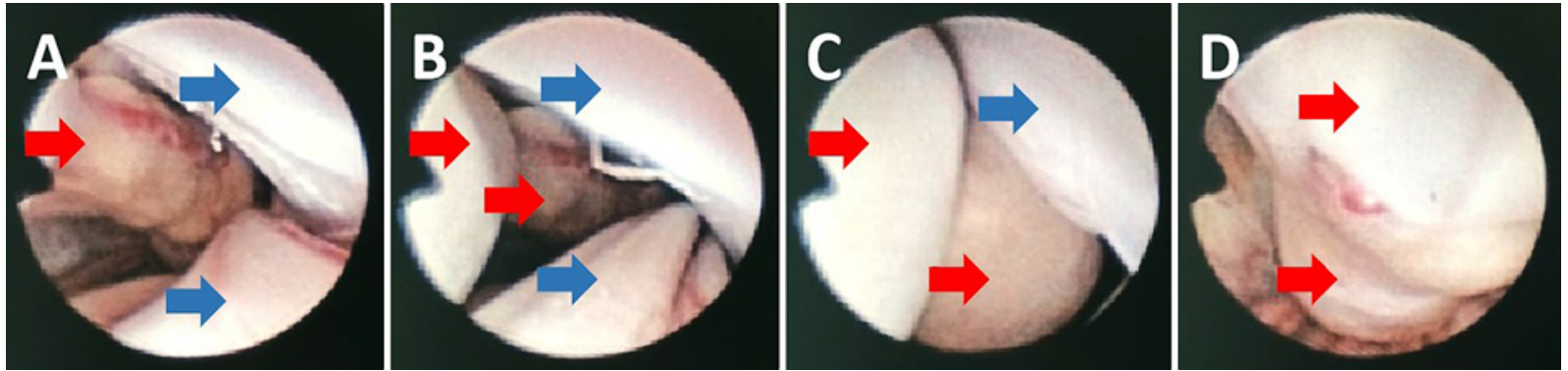

FIG. 2. Intraoperative flexible endoscopy views obtained to confirm the origin of the tumors (red arrows) and relationships with adjacent nerve roots (blue arrows). Figure is available in color online only.

end of the tumors, the exiting L5 nerve root was found to be behind the tumor, compressed but intact. Finally, the string of bead-like tumors was gently pulled out from the spinal canal via the caudal incision (Fig. 3A). A further examination was performed using the same flexible endoscope, and neither active bleeding nor tumor residual was found. Dural incisions were tightly sutured with 7-0 Prolene threads to prevent CSF leakage. Muscle and skin were closed in layers (Fig. 3B).

Pathological examination confirmed the tumors as schwannomas. The patient reported complete relief of waist and leg pain. She was discharged home 3 days after surgery. Postoperative MRI obtained at the 9-month follow-up showed no tumor residual (Fig. 1E-G). On day 3 postoperatively, VRT 3D reconstruction of lumbar CT showed bone windows created for the procedure (Fig. 1H). On clinical follow-up 9 months after the operation, the patient reported that the numbness and tingling sensations in the lower limbs were greatly relieved and there was no motor function deficit.

\section{Discussion}

Schwannomas are the most common intradural extramedullary spinal tumors, accounting for approximately $59 \%$ of all neoplasms in the spinal canal. ${ }^{1,2,5}$ Schwannoma has a favorable prognosis after total removal. Recurrence is mostly due to incomplete resection., ${ }^{1,2}$ The incidence of radicular dysfunction is low after neurectomy of tumorcarrying nerve. ${ }^{1,6}$ Bead-like schwannomas of the cauda equina are extremely rare, and are characterized by multiple schwannomas developing from a single nerve root of the cauda equina. In our current case, 10 bead-like tumors in a single string made it exceptionally rare according to our literature review. ${ }^{4}$ The beads refer to the schwannomas, and the string refers to the tumor-carrying nerve. ${ }^{3}$ Given the relatively wide intraspinal space at the lumbar segments and high mobility of the cauda equina, bead-like schwannomas of the cauda equina usually grow along the carrying nerve extensively before causing any symptoms. In our case, all the bead-like schwannomas of the cauda equina were removed by neurectomy of the branch of L5 dorsal nerve root. Our patient was instantly relieved from preoperative symptoms without additional neurological deficits at short-term follow-up.

All reported cases of bead-like schwannomas of the cauda equina, including ours, presented with nonspecific complaints such as low-back pain and radicular symptoms. ${ }^{3}$ Because these symptoms are similar to those of lumbar disc herniation, a lumbar MRI scan is strongly recommended for differential diagnosis. ${ }^{4}$ The typical MR images of bead-like schwannomas of the cauda equina are of multiple intradural nodules of various shapes and sizes in the lumbar canal, with the same signal pattern as schwannomas. ${ }^{3,4}$ Thin-layer FIESTA is useful for anatomical delineation and prognosis prediction in cranial nerve lesions, such as trigeminal neuralgia and suprasellar tumors. ${ }^{7,8}$ In our case, FIESTA was used to identify the origin of tumors and was of great value for surgical planning. Preoperative VRT 3D reconstruction of lumbar CT was also helpful in the measurement of interlaminar spaces and bone window design.

Total surgical removal is the preferred treatment for schwannoma. ${ }^{2,4,5}$ The goal of minimally invasive neu-
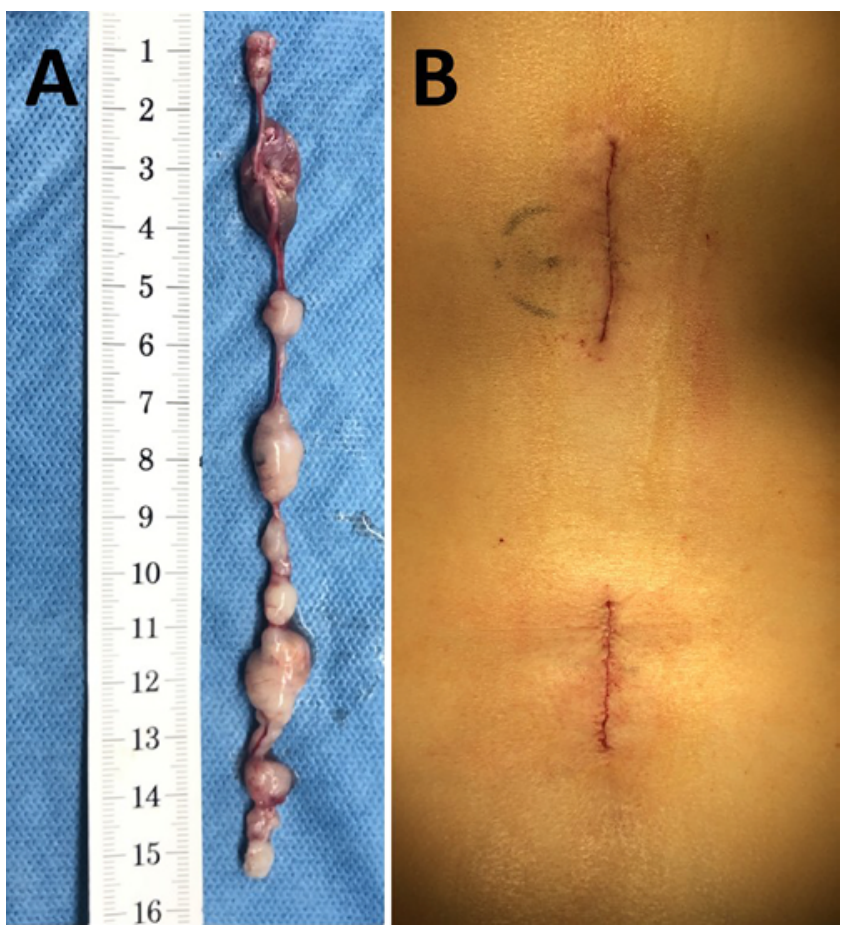

FIG. 3. A: A totally removed string of bead-like schwannomas of the cauda equina. B: Two incisions were made, at the T12 and L5 levels. Figure is available in color online only. 
rosurgery is minimizing the injury and maximizing the benefit. Conventional approaches for resection of multilevel bead-like schwannomas of the cauda equina, such as laminectomy and laminoplasty, would lead to damage of posterior column structures. ${ }^{2,4}$ Jun et al. ${ }^{3}$ reported the first case of surgical removal of bead-like schwannomas of the cauda equina. The patient underwent a laminoplasty from the L2 to S2 level, with 1-stage pedicle screw fixation to preserve the spinal stability. Liao et al. ${ }^{4}$ reported a similar case of bead-like schwannomas of the cauda equina. In that case, the tumors were detached and towed out via hemilaminectomy at 2 incision sites. However, pulling out the string of tumors without direct visualization of the tumors themselves and the cauda equina they are attached to could be quite risky.

Endoscopes are gaining popularity for resection of intraspinal tumors, and this approach has advantages such as being less invasive and leading to quicker patient recovery when compared with open surgery. ${ }^{9-11}$ The specific instance where endoscope use would be of benefit is a scenario such as the current case, in which the beads of schwannomas differ in their size and exert different space-occupying effects at different spinal levels. Sometimes they would adhere to the subarachnoid membrane and small vessels. Therefore, pulling this string of beaded schwannomas without direct visualization by an endoscope would be very risky. Nevertheless, current endoscopic equipment adopted for intraspinal tumor resection is not suitable for spinal canal exploration because of its rigid working channels. Our innovative introduction of a flexible endoscope (a soft choledochoscope) achieved the goal of revealing the anatomical relationships between tumors and nerve roots without causing further injuries. Electrophysiological monitoring is sensitive in detecting neurological damage during spinal cord surgery. ${ }^{12}$ With intraoperative electrophysiological monitoring as for our current case, tumor resection will be less "blind." Furthermore, despite providing a broad surgical exposure, hemilaminectomy would sacrifice the bony connection between the vertebral pedicle and spinous process. This bony "bridge" is well preserved in hemisemilaminectomy, and it is believed to facilitate the bone healing based on our previous experience. With a well-planned surgery based on preoperative imaging and the assistance of a flexible endoscope for visualization, our patient was successfully treated at little traumatic cost.

\section{Conclusions}

Our patient presented with 10 bead-like schwannoma nodules on a single nerve root, which made it an extremely rare case according to the literature review. Preoperative FIESTA scanning and VRT 3D reconstruction CT are of great value for diagnosis and surgical planning. With the assistance of a flexible endoscope for visualization, hemisemilaminectomy at 2 incision sites is a feasible minimally invasive approach for complete removal of beadlike schwannomas of the cauda equina. As the technology develops, the endoscopic instrument may help to improve the safety of spinal cord surgery.

\section{Acknowledgments}

This study was supported by the Science and Technology Department of Zhejiang Province (grant no. 2007C33042) and the Provincial Key R\&D Program, Science and Technology Department of Zhejiang Province (grant no. 2017C03018).

\section{References}

1. Jinnai T, Koyama T. Clinical characteristics of spinal nerve sheath tumors: analysis of 149 cases. Neurosurgery. 2005;56(3):510-515.

2. Viereck MJ, Ghobrial GM, Beygi S, Harrop JS. Improved patient quality of life following intradural extramedullary spinal tumor resection. J Neurosurg Spine. 2016;25(5):640-645.

3. Jun W, Yi-Jun K, Xiang-Sheng Z, Jing W. A long-segment string of bead-like schwannoma of cauda equina: a case report. Turk Neurosurg. 2010;20(4):540-543.

4. Liao D, Zhang J, Li D, et al. Distinctive surgical strategy for removal of string of beadlike schwannomas of cauda equina. World Neurosurg. 2018;111:207-210.

5. Naito K, Yamagata T, Nagahama A, et al. Surgical management of solitary nerve sheath tumors originating around the epiconus or conus medullaris: a retrospective case analysis based on neurological function. Neurosurg Rev. 2018;41(1):275-283.

6. Celli P. Treatment of relevant nerve roots involved in nerve sheath tumors: removal or preservation? Neurosurgery. 2002;51(3):684-692.

7. Chávez GD, De Salles AA, Solberg TD, et al. Three-dimensional fast imaging employing steady-state acquisition magnetic resonance imaging for stereotactic radiosurgery of trigeminal neuralgia. Neurosurgery. 2005;56(3):E628.

8. Watanabe K, Kakeda S, Yamamoto J, et al. Delineation of optic nerves and chiasm in close proximity to large suprasellar tumors with contrast-enhanced FIESTA MR imaging. Radiology. 2012;264(3):852-858.

9. Reisch R, Koechlin NO, Marcus HJ. Minimally invasive keyhole approaches in spinal intradural tumor surgery: report of two cases and conceptual considerations. J Neurosurg Sci. 2016;60(3):392-397.

10. Ying GY, Yao Y, Shen F, et al. Percutaneous endoscopic removal of cervical foraminal schwannoma via interlaminar approach: a case report. Oper Neurosurg (Hagerstown). 2018;14(1):1-5.

11. Zhu YJ, Ying GY, Chen AQ, et al. Minimally invasive removal of lumbar intradural extramedullary lesions using the interlaminar approach. Neurosurg Focus. 2015;39(2):E10.

12. Hadley MN, Shank CD, Rozzelle CJ, Walters BC. Guidelines for the use of electrophysiological monitoring for surgery of the human spinal column and spinal cord. Neurosurgery. 2017;81(5):713-732.

\section{Disclosures}

The authors report no conflict of interest concerning the materials or methods used in this study or the findings specified in this paper.

\section{Author Contributions}

Conception and design: Zhu. Acquisition of data: Zhao, Shen. Analysis and interpretation of data: Xie, Ying. Drafting the article: Ren, Shen. Administrative/technical/material support: Xie.

\section{Correspondence}

Yong-Jian Zhu: Second Affiliated Hospital of Zhejiang University School of Medicine, Hangzhou, Zhejiang, China. neurosurgery@ zju.edu.cn. 